\title{
VALUTAZIONE DEL SISTEMA DIAGNOSTICO NUCLISENS EASYQ PER LA QUANTIZZAZIONE DELLA CARICA VIRALE HIV-I
}

Ventura A., Bruzzone B., Caligiuri P., Nigro N., Frabetti M., Icardi G.

\section{Dipartimento di Scienze della Salute,} Università degli Studi di Genova

Introduzione: La determinazione quantitativa della carica virale HIV assieme al numero dei linfociti CD4+ è fondamentale per seguire l'evoluzione clinica del paziente sieropositivo. Le nuove tecnologie mettono a disposizione strumenti sempre piu' sensibili e sofisticati. Nel presente studio sono state valutate le performance del sistema Nuclisens EasyQ (Biomerieux), che combina l'amplificazione NASBA con la rilevazione in real-time con sonde molecular beacon, rispetto al metodo NucliSens HIV1-QT usato in precedenza. Metodo: Sono stati analizzati 60 campioni di plasma provenienti da pazienti in terapia antiretrovirale, la cui carica virale era gia' stata quantificata con il test NucliSens HIV1-QT dopo estrazione semiautomatica, SAME, su MINIMAG. I campioni saggiati ricoprono l'intero range di viremia che si puo' riscontrare routinariamente nei pazienti. Sono state effettuate prove di riproducibilità intrasaggio ed intersaggio. Sono stati inoltre saggiati anche 5 sottotipi non-B per testare l'efficienza della metodica in questi casi particolari.

Risultati: Nell'87\% (52/60) dei campioni analizzati si sono rilevate differenze inferiori a $0.5 \mathrm{log}$, convenzionalmente considerata soglia di accettabilità per l'equivalenza dei risultati. I risultati dei 5 sottotipi non-B sono stati confrontati con 
le due metodiche utilizzate nella routine (Nuclisens HIV-1 QT e Branched Bayer) e si sono ottenuti risultati sovrapponibili a quelli ottenuti con il sistema Bayer. Per quanto riguarda le prove di riproducibilità le differenze tra le due metodiche sono risultate inferiori a $0.2 \log$ e comprese tra 0.2 e 0.5 log rispettivamente per le prove di riproducibila' intersaggio e intrasaggio.

Conclusioni: La metodica esaminata ha fornito risultati soddisfacenti sia per la sovrapponibilita' dei risultati con la precedente metodica sia per la riproducibilita' intersaggio ed intrasaggio. Inoltre a differenza dal sistema NucliSens HIV1QT, il test oggetto dello studio e' stato in grado di rilevare la carica virale di campioni con sottotipi HIV-1 non-B. 